\title{
LOCAL TRANSVERSE COUPLING CONTROL
}

\section{O. Dallin, Canadian Light Source, University of Saskatchewan, 107 North Road, Saskatoon SK, S7N 5C6, Canada}

\begin{abstract}
The brightness of light produced at a point (s) in a light source is inversely proportional to $\psi^{\mathrm{n}}(\mathrm{s}) \mathrm{f}(\beta)$, where $\psi(\mathrm{s})$ is the local coupling angle, $f(\beta)$ is a function of the transverse beta-functions and $\mathrm{n}$ depends on photon diffraction effects and ranges from 1 to 2 . As well, brightness is proportional to the circulating current, which, for a given lifetime, is proportional to the average vertical beam size and hence to the average coupling angle, $\psi_{\text {av }}$, over the entire lattice. At a given point, then, brightness is proportional to $\psi_{\mathrm{av}} /\left[\psi^{\mathrm{n}}(\mathrm{s}) \mathrm{f}(\beta)\right]$. Simulations performed on the Canadian Light Source (CLS) lattice indicate that it is possible to decrease the coupling locally resulting in enhancements of the local brightness by factors of 3 to 8 . This is achieved with an array of independent skew quadrupoles. Corrections are applied using the Singular Value Decomposition (SVD) algorithm to adjust the coupling angle. A practical beam-coupling monitor as suggested by Teng has also beam demonstrated. Other techniques for controlling local coupling are also discussed. All techniques are well suited for global coupling control as well.
\end{abstract}

\section{INTRODUCTION}

This report describes techniques to reduce the local coupling, $\psi(\mathrm{s})$, while the average coupling, $\psi_{\mathrm{av}}$, remains large to preserve beam lifetime. Consequently, for a given lifetime or average coupling, the brightness is enhanced where the local coupling is decreased. Local enhancements approaching a factor of ten have been simulated.

\subsection{Coupling}

Following Teng[1], coupling is considered to vary locally as a function of position (s) around the lattice. The local coupling angle, $\psi(\mathrm{s})$, and the (invariant) transverse emittance, $\varepsilon$, determine the local horizontal and vertical emittances:

$$
\varepsilon_{\mathrm{x}}(\mathrm{s})=\cos ^{2} \psi(\mathrm{s}) \varepsilon ; \varepsilon_{\mathrm{y}}(\mathrm{s})=\sin ^{2} \psi(\mathrm{s}) \varepsilon ; \varepsilon=\varepsilon_{\mathrm{x}}(\mathrm{s})+\varepsilon_{\mathrm{y}}(\mathrm{s})
$$

In the following, small coupling angles are considered, so $\sin \psi \approx \psi$ and $\varepsilon_{\mathrm{x}}(\mathrm{s}) \approx \varepsilon$,

\subsection{Brightness}

Brightness for various regimes of relative photon diffraction is described by Kim[2]. For high energy machines ( $>6 \mathrm{GeV})$, optimized for hard x-rays, brightness, $\boldsymbol{B}_{0}$, is emittance dominated and at position (s) is given by:

$$
\begin{aligned}
{\mathfrak{B}_{0}}^{-1}(\mathrm{~s}) & \propto \varepsilon_{\mathrm{y}}(\mathrm{s}) \varepsilon_{\mathrm{x}}(\mathrm{s}) \\
& \propto \psi^{2}(\mathrm{~s})
\end{aligned}
$$

In this case, reducing the coupling locally can result in a significant increase in the local brightness inversely proportional to the square of the coupling angle.

For medium energy machines (1-3 GeV), optimized for VUV and soft $\mathrm{x}$-rays, brightness is given by:

$$
\begin{aligned}
\boldsymbol{B}_{0}{ }^{-1}(\mathrm{~s}) & \propto\left[\varepsilon_{\mathrm{x}}(\mathrm{s}) \varepsilon_{\mathrm{y}}(\mathrm{s}) \beta_{\mathrm{x}}(\mathrm{s}) \beta_{\mathrm{y}}(\mathrm{s})\right]^{1 / 2} \\
& \propto \psi(\mathrm{s})\left[\beta_{\mathrm{x}}(\mathrm{s}) \beta_{\mathrm{y}}(\mathrm{s})\right]^{1 / 2},
\end{aligned}
$$

where $\beta_{\mathrm{x}}$ and $\beta_{\mathrm{y}}$ are the horizontal and vertical machine functions. Here, the brightness is inversely proportion to the beam size, $\sigma=(\beta \varepsilon)^{1 / 2}$. For corrections where changes in the machine functions are small the brightness is inversely proportional to the coupling angle.

\subsection{Lifetime}

The beam lifetime, $\mathbf{L}$, is proportional to the average beam size around the lattice. For small coupling angles, $\mathbf{L} \propto \psi_{\text {av }}$. The beam lifetime is increased by increasing the average coupling angle.

\section{LOCAL COUPLING CONTROL}

Three techniques have been developed to reduce the coupling at some local positions in the lattice, i.e., the vertical beam size is reduced. All the techniques follow the same general procedure. Consider a lattice with $\boldsymbol{i}$ positions to be adjusted and $\boldsymbol{j}$ skew quadrupoles (skews) used to adjust the coupling. At each position, $\boldsymbol{i}$, the response, $\mathrm{S}_{i j}$, due to exciting skew $\boldsymbol{j}$ to some value $\mathrm{k}_{j}$ is measured. The skew values should be chosen so that each response is fairly linear. Each response measures the change of some function of the local coupling, $\mathrm{f}\left(\psi_{i}\right)$. To change $\mathrm{f}\left(\psi_{i}\right)$ at all $i$ locations all skews are used. The final value of the function is given by

$$
\mathrm{f}\left(\psi_{i}\right)\left(\mathrm{F}_{i}-1\right)=\mathrm{S}_{i j} \mathrm{k}_{j}
$$

where $\mathrm{F}_{i}$ is can be any desired value. E.g., for zero coupling $\mathrm{F}_{i}=0$, to double existing coupling $\mathrm{F}_{i}=2$. By choosing different values for $\mathrm{F}_{i}$ at different locations, $\mathrm{f}\left(\psi_{i}\right)$ can be adjusted locally. The skew values are given by 


$$
\mathrm{k}_{j}=\mathrm{S}^{-1}{ }_{i j}\left(\mathrm{~F}_{i}-1\right) \mathrm{f}\left(\psi_{i}\right)
$$

That is, the skew values are determined by inverting the response matrix and adjusting the values through $\mathrm{F}_{i}$. For this purpose the SVD algorithm is very useful. By restricting the larger singular values it is possible to arrive at a solution where the skew values still produce fairly linear responses in changing the value of $\mathrm{f}\left(\psi_{i}\right)$.

Three possible functions can be used. In each case $\mathrm{f}\left(\psi_{i}\right) \propto \psi_{i}$. The functions are vertical position, vertical size and coupling angle. Details are given below.

\section{SIMULATIONS}

Local coupling correction has been simulated in the CLS lattice[3] using DIMAD[4]. Misalignments and multipole errors were introduced into the lattice resulting in coupling angles around the lattice of about $100 \mathrm{mrad}$. That is, the vertical emittance was about $1 \%$ of horizontal emittance. Three techniques for local coupling control were simulated.

The function, $\mathrm{f}\left(\psi_{i}\right)$, in centre of the twelve CLS straights were measured and the response function using twelve skews were calculated. Skew values of $0.005 \mathrm{~m}^{-2}$ (0.048 T/m @ $2.9 \mathrm{GeV})$ were used.

Obtaining a useful result where the local coupling varied significantly took several trials. Some larger "singular values", produced using SVD to invert the response function matrix, had to be omitted from the solution in order that the skew values were neither too small (no response) nor too large (non-linear response). Adjusting the factors, $F_{i}$, to produce a smooth variation from cell to cell was also required. After some manipulation is was possible to adjust the local coupling so that minimum coupling at some point was less than half the average coupling.

\subsection{Vertical Size}

In this case the vertical sizes, $\sigma_{i}$, are measured at $i$ locations. Using the same skew values, $\Delta \mathrm{k}_{j}$, at $j$ locations and activating one skew at a time, new sizes, $\sigma_{i j}$, are measured. The response matrix is given by $S_{i j}=\Delta \sigma_{i j} / \Delta \mathrm{k}_{j}$, where $\Delta \sigma_{i j}=\sigma_{i j}-\sigma_{i}$.

With the skew values mentioned, changes in vertical beam size, $\Delta \sigma_{i j}$, are typically a few microns. Measuring the beam size to this accuracy at positions of interest makes this technique difficult in practice. Never the less, since the correction is acting directly on the beam size, this technique is best suited to machines where the brightness is inversely proportional to beam size, i.e., to intermediate energy machines. Beam size measurements aside, simulations indicate that in should be possible to enhance the brightness by more than a factor of two at some positions in the lattice.

\subsection{Coupling Angle}

In this case the coupling angles, $\psi_{i}$, are measured at $i$ locations and then again with the skews turned on one at a time. Changes in coupling angle, $\Delta \psi_{i j}=\psi_{i j}-\psi_{i}$, are measured. The response is given by $S_{i j}=\Delta \psi_{i j} / \Delta k_{j}$.

This technique depends on measuring the local coupling angle. This will be discussed in section 4 . It requires measuring beam position changes of tens of microns. Since the correction acts directly on the coupling angle and consequently the emittance, this technique does not necessarily reduce the beam size and is best suited for machines where brightness is inversely proportional to emittance, i.e., the high energy machines.

Three examples of reduced emittance are shown in Figure 1. At one extreme the emittance is smallest but the beam size, $\sigma$, is largest. At this extreme the machine parameters are $\varepsilon_{\mathrm{y}}=0.004 \mathrm{~nm}-\mathrm{rad}, \beta_{\mathrm{y}}=510 \mathrm{~m}$ and $\alpha_{\mathrm{y}}=$ 75! The smallest beam size has the largest emittance: $\varepsilon_{\mathrm{y}}=$ $0.03, \beta_{\mathrm{y}}=1.1$ and $\alpha_{\mathrm{y}}=-0.12$. The intermediate ellipse has $\varepsilon_{\mathrm{y}}=0.02, \beta_{\mathrm{y}}=33$ and $\alpha_{\mathrm{y}}=-0.54$. In the low emittance case the coupling correction has radically changed the beam symmetry $(\alpha=0)$ and produced a vertical emittance that is extremely skewed.

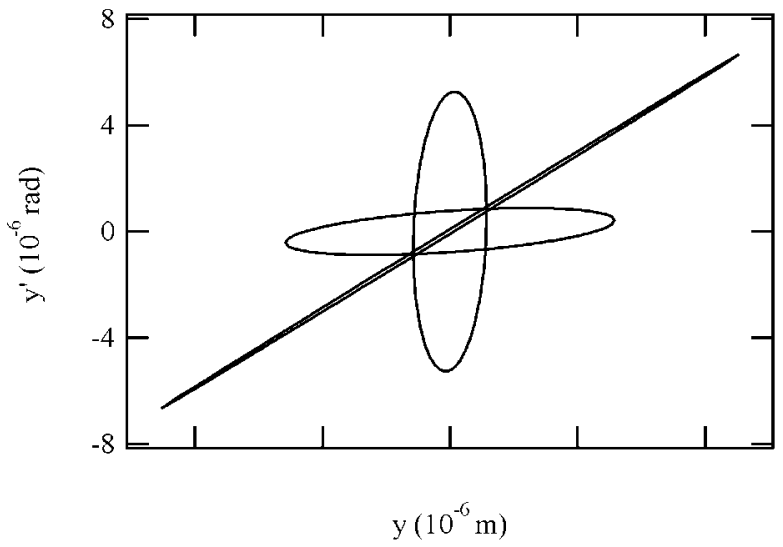

Figure 1. Examples of the vertical emittance produced by reducing the local coupling.

This technique could be used for both intermediate and high energy machines.

\subsection{Vertical Position}

This technique probes betatron oscillations created by a pulsed horizontal kick. While the beam is oscillating, vertical positions, $\mathrm{y}_{i}$, are measured at $\boldsymbol{i}$ locations and then again with the skews turned on one at a time. The response matrix is given by $\mathrm{S}_{i j}=\Delta \mathrm{y}_{i j} / \Delta \mathrm{k}_{j}$, where $\Delta \mathrm{y}_{i j}=\mathrm{y}_{i j}$ $-y_{i}$. This technique is the easiest to implement since the vertical position is relatively easy to measure.

With the skew values mentioned, changes in vertical beam position, $\Delta \mathrm{y}_{i j}$, are typically tens of microns. Since this correction is acting neither on the beam size or emittance the results vary between reducing the emittance and reducing the beam size, although extremely skewed 
emittances were not observed. Consequently this technique could be useful for either intermediate or high energy machines.

\subsection{Local Brightness}

Results of the local brightness enhancement are shown in Figure 2. Results from all three techniques are plotted. For the position and size techniques the brightness is assumed to depend on the beam size. These are the two low lying plots. For the coupling angle technique the brightness is assumed to depend on the beam emittance only. For this the results are those that produced the intermediate ellipse in Figure 1. (Note: although the corrections were performed on an intermediate energy machine, it is assumed that the corrections would work on any machine.) The dashed line represents the relative brightness for a lattice with uniform coupling and the same lifetime, normalized to unity. It appears that for medium energy machines local enhancements of a factor of two or more are attainable. For high energy machines the enhancement approaches a factor of eight.

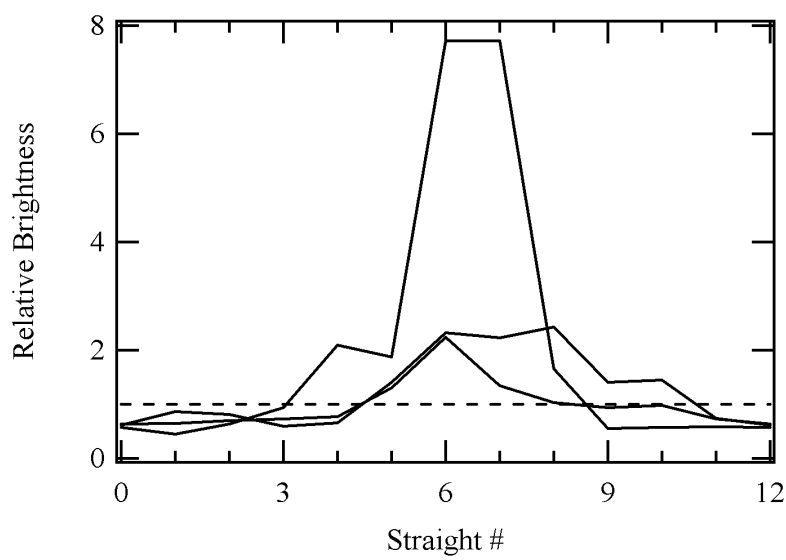

Figure 2. Simulated relative local brightness at the centre of each of the twelve CLS straights from three different coupling correction schemes.

\subsection{Global Orbit Control}

The local coupling correction schemes are all very useful for applying a global coupling correction. For this purpose the scaling factors, $F_{i}$, are all set to the same value. In this way the coupling can be made larger or smaller as desired. Another way to get a global correction is to adjust the coupling at one location only. In this case, the coupling tends to be the same at all locations. In either case the machine functions are relatively undistorted.

\section{BEAM COUPLING MONITOR}

Teng[1] describes a beam coupling monitor (BCM) and shows that the coupling angle can be determined by monitoring the vertical response, $\left[\delta y, \delta y^{\prime}\right]$, to two linearly independent horizontal perturbations of the beam applied at the location of the monitor. The two perturbations have the form $[\delta \mathrm{x}, 0]$ and $\left[\delta \mathrm{x}^{\prime}, 0\right]$ and can be created by two horizontal orbit correctors. The angular perturbation is easily produced by a corrector at the location of the monitor. The dislocation, $[\delta x, 0]$, can be produced by another corrector near the first, while the first is used to remove the angular component. It can be shown that eq. (17) of reference [1] is approximately satisfied if the two correctors are close together, in any order, and the coupling components of the transfer matrix between the two are small.

The BCM requires the measure of $\delta \mathrm{x}, \delta \mathrm{x}^{\prime}, \delta \mathrm{y}$ and $\delta \mathrm{y}^{\prime}$ for both of the perturbations. While the displacements are obtained from the position monitor, the angular changes are obtained by employing a second monitor near the first.

Simulations show that the coupling angles measured with the BCM are in close agreement with the values calculated directly in DIMAD. Response functions were in even better agreement since they are measuring changes in coupling angle.

For practical purposes the perturbations must be large enough to produce vertical shifts of several microns. In the simulations, corrector values around $0.5 \mathrm{mrad}$ were sufficient to produce vertical shifts up to 20 microns.

\section{DISCUSSION}

Coupling values have been verified by tracking. DIMAD calculates the principal lattice functions[1] for the coupled beam ( $\mathrm{u}, \mathrm{v}$ space). Transformations to real space ( $x, y$ space) must be made to produce the results shown in Figure 1. More details are given in reference [5]. This paper and the modified version of DIMAD are available by contacting the author: les@cls.usask.ca.

The work to date stands as a proof of principal. More detailed work to include effects of energy spread and measurement errors is required. CLS will have 36 independent skew quadrupoles. Studies implementing all the correctors will be done to see if the local coupling control can be improved.

At the very least, local coupling control provides a straight forward way to control global coupling. Beyond that, local coupling correction is a viable technique for enhancing the brightness at some points in a lattice while preserving beam lifetime. Different light users have different brightness requirements and it may be possible to tailor the beam to suit a variety of needs.

\section{REFERENCES}

[1] L C. Teng, "Global and Local Horizontal-Vertical Decoupling”, PAC'97, Vancouver, p. 1359.

[2] K. Kim, "Characteristics of Synchrotron Radiation", AIP Conference Proceedings 184, p. 565, 1989.

[3] L. O. Dallin, "Canadian Light Source: An Update", this proceedings.

[4] R. V. Servranckx, DIMAD, (updated Oct. 2000).

[5] L. O. Dallin and R. V. Servranckx, "Local Transverse Coupling Control", CLS Note 5.7.36.1, Oct. 23, 2000. 